\title{
Calcaneal Lengthening For Correction of Symptomatic Flexible Flat Foot in Children
}

\author{
Elshafey A. Elshafey ${ }^{a}$, Mohamed O. Hegazy ${ }^{b}$, Hani A. Bassiouni ${ }^{b}$, Mohamed E. Al-Ashhab ${ }^{\text {b }}$
}

Department of orthopedic, $6^{\text {th }}$ october health insurance hospital, Egypt. ${ }^{\mathrm{b}}$ department of orthopedic, Faculty of Medicine, Benha University, Egypt

Correspondence to: Elshafey A. Elshafey, Department of orthopedic, $6^{\text {th }}$ october health insurance hospital, Egypt

Email:

dr_elshafey2005@yahoo.com

Received::21 July 2019

Accepted: 9 October 2019

\begin{abstract}
:
Background; The purpose of this study was to evaluate the clinical, functional and radiological outcome of calcaneal lengthening osteotomy for the treatment of symptomatic flatfoot deformity in children.
\end{abstract}

Methods; A prospective multicentre study on 29 feet of 23 patients who underwent lateral calcaneal lengthening for symptomatic flatfoot in children in the period between October 2015 and June 2017. Inclusion criteria were symptomatic flexible flatfoot in children with failed non operative treatment. During the study period, 29 feet of 23 patients with symptomatic flatfoot underwent calcaneal lengthening osteotomy. All patients met the inclusion criteria and were available for clinical and radiological follow-up. The percentage male were $(62 \%)$-female were (38\%), The mean age at time of surgery was 10.4 years old ( 7 - 17 years old) and their mean follow up period was 26.2months.The mean functional American Orthopaedic Foot and Ankle Society (AOFAS) Ankle-Hindfoot score had improved from 43.45 (31-55) preoperatively to $85.52(68-92)$ postoperatively at last follow up ( $\mathrm{P}$ value $<0.001)$ using paired t-test as a significance test for evaluation of pre and post-operative AOFAS score

Conclusions; The results of this study support the combination of lateral column lengthening and soft tissue reconstruction for treatment of Plano-valgus foot deformity, The adopted technique revealed satisfactory result both in children and adolescents.

Keywords: Flatfoot, Symptomatic, Calcaneal lengthening, Surgery, children 


\section{Introduction:}

Approximately 20 percent of all musculoskeletal complaints are related to the foot and ankle, Flatfoot is the term used to describe a weight-bearing foot shape in which the hindfoot is in valgus alignment, the midfoot sags in a planter direction with reversal of the longitudinal arch, and the forefoot is supinated in relation to the hindfoot ${ }_{(1)}$. Flatfoot may exist as an isolated pathology or as part of a larger clinical entity.

Paediatric flatfoot can be divided into flexible and rigid categories. Flexible flatfoot is characterized by a normal arch during nonweight bearing and a flattening of the arch on stance. The arch elevates spontaneously in most children during the first decade of life. Flexible flatfoot may be asymptomatic or symptomatic $_{(2) .}$

Symptomatic forms of flexible flatfoot produce subjective complaints, alter function, and produce significant objective findings. When all nonsurgical treatment options have been exhausted, surgical intervention can be considered $_{(2)}$.

Options for surgical treatment vary from simple soft tissue procedures to calcaneal osteotomy, subtalar extra-articular arthroresis and triple arthrodesis (3).

The purpose of this study was to evaluate the short term results of calcaneal lengthening osteotomy in the treatment of symptomatic flexible flatfoot.

\section{Patients And Methods:}

A prospective multicentre study was done on 29 feet of 23 patients who underwent lateral calcaneal lengthening for symptomatic flatfoot in the period between October 2015 and June 2017. The mean age was 10.4 years old (7 - 17 years) and their mean follow up period was 26.2 months. Inclusion criteria were symptomatic flexible flatfoot in children with failed non- operative treatment. Exclusion criteria include rigid flatfoot, arthritic changes, tarsal coalition, rheumatoid arthritis, post traumatic, tibialis posterior tendon dysfunction, neuromuscular disorders. Each patient in this study was carefully assessed radiologically and clinically by taking a detailed clinical history and examination and adequate information from the child or parent to determine whether the flat foot deformity is painful or pain free.

Demographic and clinical data were recorded as follows: gender, underlying pathology, age at time of surgery, side operated, details of surgical procedure, any concomitant procedures performed and length of followup.

\section{Operative Procedure:}

All procedures were performed under general anaesthesia. The patients operated supine on a 
radiolucent table, pneumatic thigh tourniquet was used. A modified lateral longitudinal Ollier incision about $3 \mathrm{~cm}$ that extended $1 \mathrm{~cm}$ proximal to anterior calcaneal process was made over sinus tarsi, Calcaneocuboid joint was identified, the interval between middle and anterior facets of calcaneus was identified

A second longitudinal incision along the medial border of the foot was made. The tibialis posterior tendon was cut in a Zmanner. A talonavicular capsulotomy was made, Percutaneous Achilles tendon lengthening was performed in all patients (fig.1).

An oblique calcaneal osteotomy was done between the anterior and middle facets about $2 \mathrm{~cm}$ posterior to the calcaneocuboid joint (fig. 2).A Kirschner wire was inserted from the cuboid distally across the calcaneocuboid joint to avoid its subluxation with osteotomy distraction and iliac crest bone graft was placed through the opened osteotomy then advancing the calcaneocuboid k-wire to fix the graft (fig. 1).

Medial and plantar capsular imbrication of the talonavicular joint, was performed. The proximal slip of the tibialis posterior was advanced about 5 to $7 \mathrm{~mm}$ through a slit in the distal stump of the tendon and repaired. Fixation of advanced dorsal slip of tibialis posterior tendon through tunnel into navicular bone was done in 10 patients $(10 / 29,34.5 \%)$, while in 19 patients fixation by direct suturing of the dorsal slip to the periosteum of navicular bone combined with interosseous sutures $(19 / 29,65.5 \%)$, was applied. The incisions were closed, and a well-padded short-leg non-weight-bearing cast was applied (fig. 1)

Antero-posterior and lateral X-rays for the operated foot, was done on the same day of operation.

Non-weight bearing below knee cast for the 1st 6 weeks post-operatively had been done. At $6^{\text {th }}$ week pins and cast are removed, protective weight bearing as tolerated by the patients allowed. Return to pre-operative function and activity as tolerated by the patients as early as possible to be tried.

From the 6th week to 6-month postoperative patients used medial longitudinal arch support.

At the final follow-up, the outcome was assessed clinically by the AOFAS ankle-hind foot scale and by radiographic parameters.

\section{Statistical analysis:}

Data were statistically described in terms of mean standard deviation (SD), median and range. Comparison between pre- and postoperative values was done using paired $t$ test. $p$ values less than 0.05 was considered statistically significant. All statistical calculations were done using computer 
program IBM SPSS (Statistical Package for the Social Science) release22for Microsoft
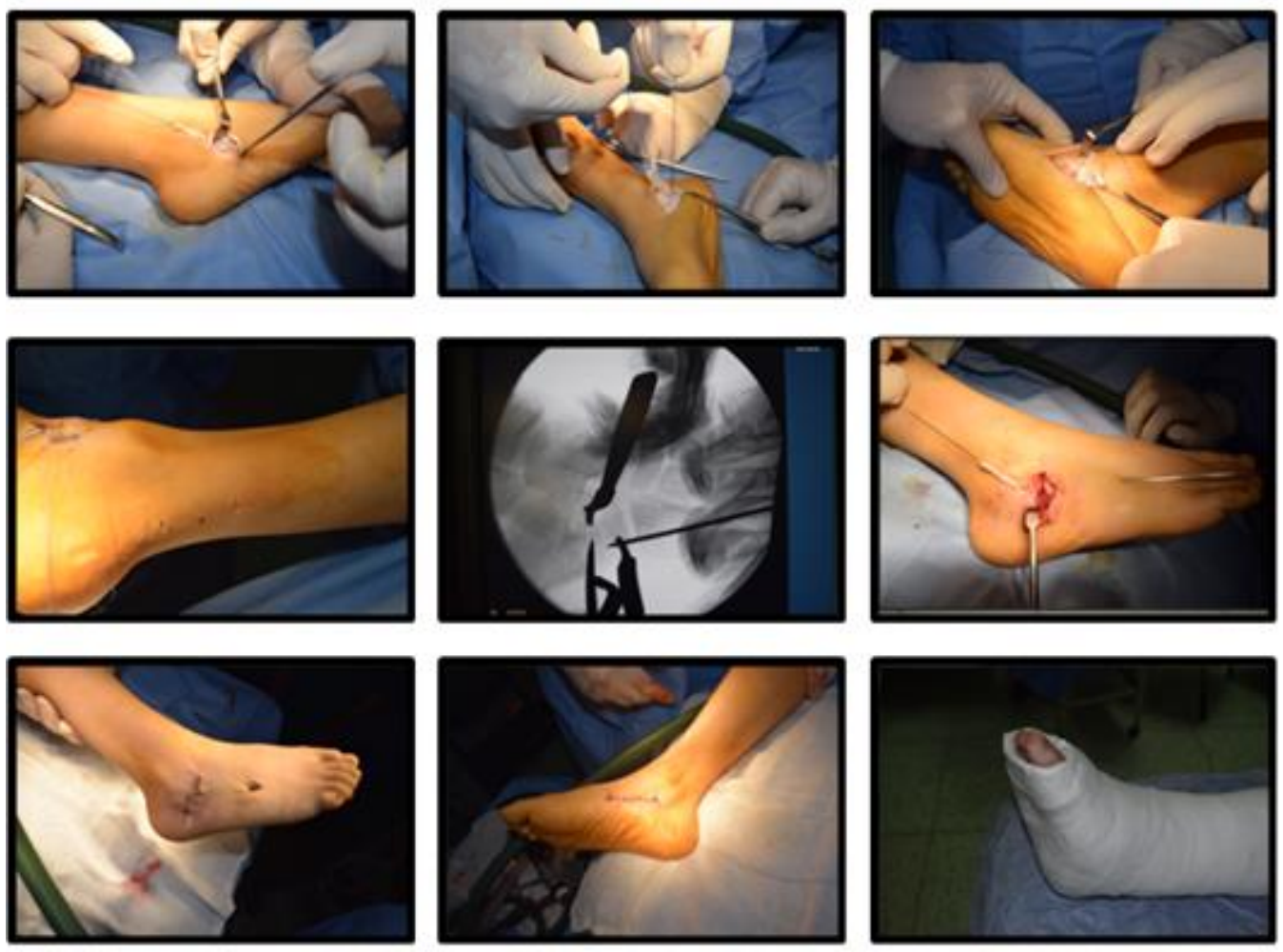

Fig. 1: the operative procedure

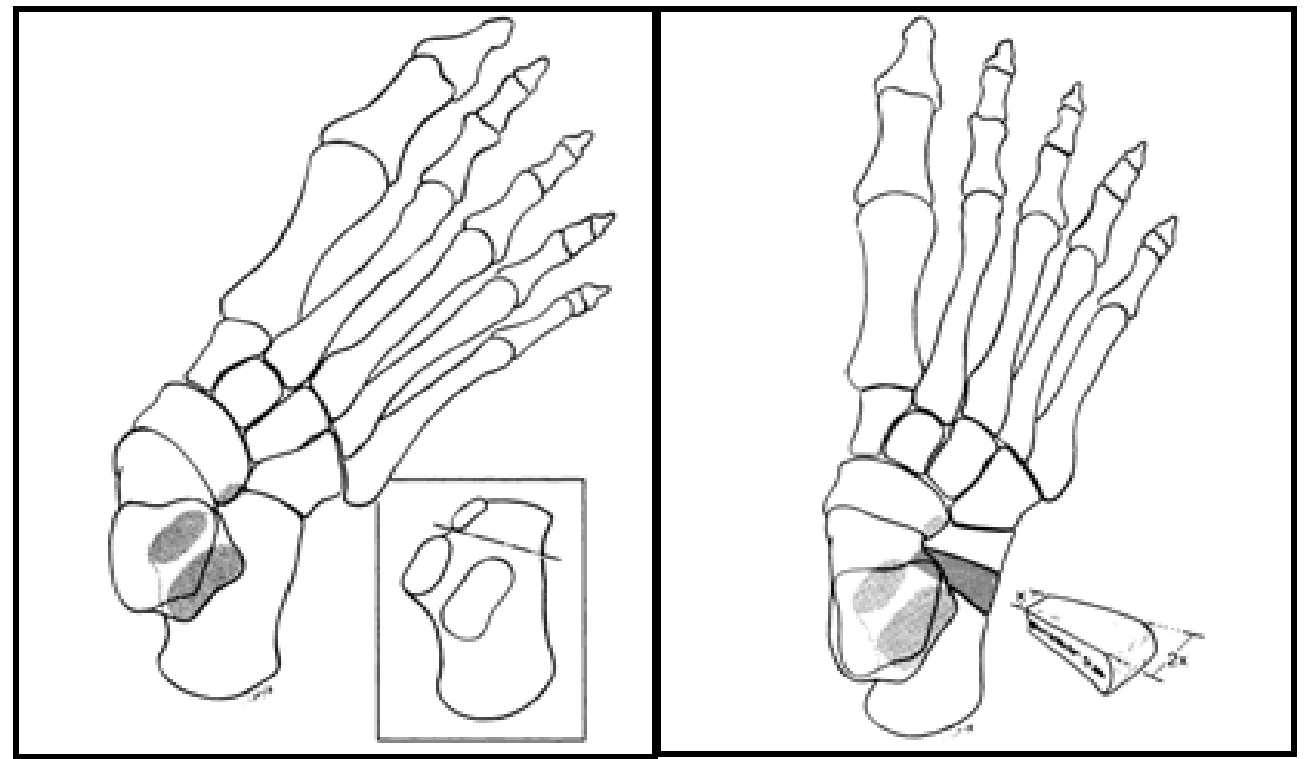

Fig.2: Dorsal view showing correction of all components of the deformity with the trapezoid-shaped graft in place 


\section{Results:}

During the study period, 29 feet of 23 patients with symptomatic flatfoot underwent calcaneal lengthening osteotomy. All patients met the inclusion criteria and were available for clinical and radiological follow-up (Table 1). The male-female percentage was $(62 \%$, $38 \%)$. Mean age at time of surgery was (10.4 years).

The mean functional American Orthopaedic Foot and Ankle Society (AOFAS) AnkleHindfoot score improved from 43.45 (31-55) preoperatively to 85.52 postoperatively at last follow up ( $\mathrm{P}$ value < 0.001) using paired t-test as a significance test for evaluation of pre and post-operative AOFAS score.

\section{Radiological results}

Pre and post-operative radiological scoring (Table 1)

All bone grafts united with the calcaneus within an average of 2 months, complete incorporation and remodelling occurred within 6 months, and there was no complication regarding the graft site. There was no dorsal subluxation at the level of calcaneo-cuboid joint postoperatively. Postoperative complications were seen in 2 patients $(6.9 \%), 1$ patient $(3.45 \%)$ had postoperative superficial wound infection on the
Table (1): Pre and post operative radiological scoring using AP TNA and Lat. TMTA.

\begin{tabular}{|c|c|c|c|c|c|c|c|}
\hline \multirow[t]{2}{*}{ Number } & \multirow[t]{2}{*}{ age } & \multirow[t]{2}{*}{ sex } & \multirow[t]{2}{*}{ side } & \multicolumn{2}{|c|}{ pre } & \multicolumn{2}{|c|}{ Post } \\
\hline & & & & TNA & TMTA & TNA & TMTA \\
\hline $1-1$ & 7 & $\widehat{\sigma}$ & $\mathrm{Lt}$ & 44 & 35 & 8 & 3 \\
\hline $1-2$ & & & Rt & 40 & 32 & 7 & 3 \\
\hline $2-3$ & 13 & 우 & $\mathrm{Lt}$ & 15 & 12 & 5 & 0 \\
\hline $3-4$ & 13 & 우 & $\mathrm{Rt}$ & 20 & 22 & 3 & 0 \\
\hline $3-5$ & & & $\mathrm{Lt}$ & 17 & 15 & 5 & 0 \\
\hline $4-6$ & 9 & $\sigma^{\lambda}$ & Rt & 37 & 35 & 10 & 0 \\
\hline $4-7$ & & & $\mathrm{Lt}$ & 28 & 30 & 18 & 10 \\
\hline $5-8$ & 11 & q & Rt & 35 & 30 & 7 & 3 \\
\hline $5-9$ & & & $\mathrm{Lt}$ & 40 & 35 & 7 & 3 \\
\hline $6-10$ & 7 & o & Rt & 70 & 40 & 20 & 10 \\
\hline $7-11$ & 14 & a & $\mathrm{Rt}$ & 40 & 18 & 8 & 0 \\
\hline $8-12$ & 16 & a & $\mathrm{Lt}$ & 15 & 30 & 7 & 8 \\
\hline $9-13$ & 16 & q & Rt & 12 & 26 & 3 & 0 \\
\hline $10-14$ & 11 & $0^{\pi}$ & $\mathrm{Lt}$ & 20 & 20 & 4 & 0 \\
\hline $11-15$ & 12 & $0^{\pi}$ & Rt & 40 & 35 & 8 & 4 \\
\hline $12-16$ & 7 & 우 & $\mathrm{Rt}$ & 20 & 45 & 8 & 10 \\
\hline $13-17$ & 11 & $\sigma^{\pi}$ & $\mathrm{Lt}$ & 60 & 35 & 10 & 3 \\
\hline $14-18$ & 11 & q & Rt & 30 & 22 & 5 & 8 \\
\hline $14-19$ & & & $\mathrm{Lt}$ & 22 & 20 & 5 & 5 \\
\hline $15-20$ & 14 & $0^{\pi}$ & $\mathrm{Lt}$ & 40 & 35 & 7 & 5 \\
\hline $16-21$ & 13 & q & $\mathrm{Lt}$ & 32 & 15 & 5 & 0 \\
\hline $17-22$ & 16 & $0^{\pi}$ & Rt & 33 & 20 & 7 & 3 \\
\hline $18-23$ & 13 & $0^{\pi}$ & $\mathrm{Lt}$ & 18 & 25 & 3 & 3 \\
\hline $18-24$ & & & Rt & 15 & 20 & 3 & 0 \\
\hline $19-25$ & 12 & o & $\mathrm{Lt}$ & 62 & 45 & 10 & 8 \\
\hline $20-26$ & 12 & $0^{\pi}$ & $\mathrm{Lt}$ & 60 & 38 & 15 & 15 \\
\hline $21-27$ & 15 & $0^{\pi}$ & Rt & 18 & 15 & 3 & 0 \\
\hline $22-28$ & 17 & $0^{\lambda}$ & $\mathrm{Lt}$ & 20 & 16 & 3 & 3 \\
\hline $23-29$ & 13 & a & $\mathrm{Rt}$ & 25 & 14 & 5 & 0 \\
\hline
\end{tabular}

lateral aspect of the foot after 2 weeks, the patient received antibiotic and frequent dressing till infection subsided and wound healed within 4 weeks monitored by 
normalized ESR and CRP. Recurrence of tendoachiles contracture after surgery in one patient (3.45\%), stretching exercises was done till complete improvement without any need to surgical intervention.

\section{Case (Female patient $15 y$ old with bilateral}

flexible flatfoot): (fig $3 \& 4$ )
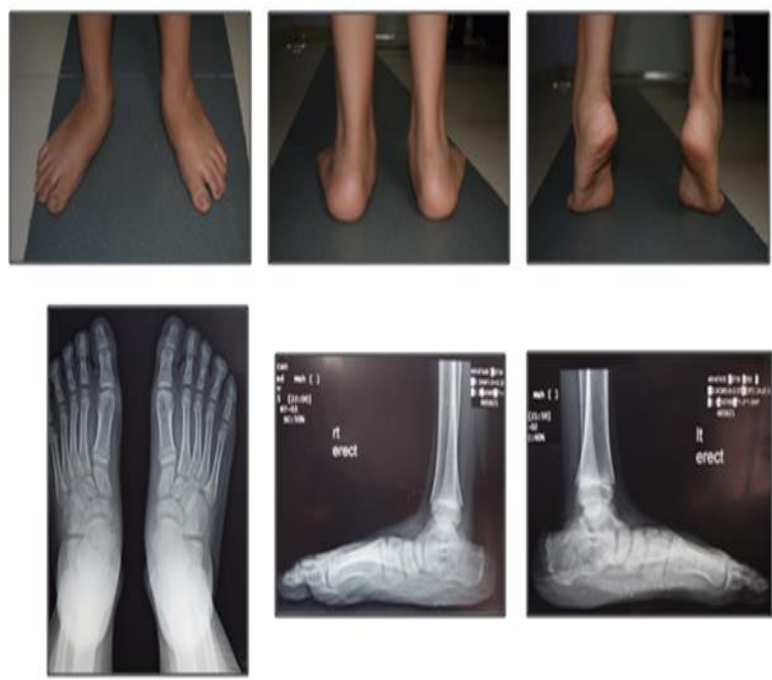

Fig. 3: Pre operative photos of a 15 y old female patient
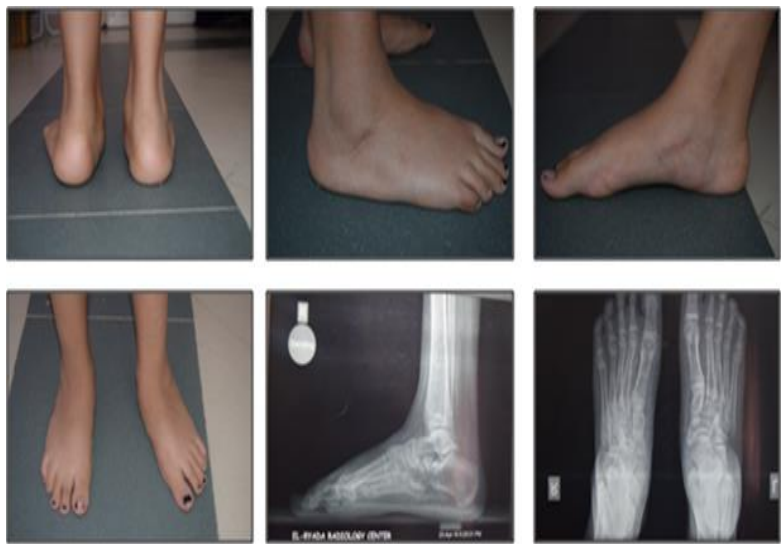

Fig. 4: Post operative photos

\section{Discussion:}

Many options for surgical treatment have been proposed for the correction of flatfoot deformity. The surgical options vary from simple soft tissue procedures to calcaneal osteotomy, subtalar extra-articular arthrodesis and triple arthrodesis. It has been widely recognized that isolated soft tissue procedures do not provide stable correction of the deformity, which often progresses and becomes painful. For this reason, bony osteotomies have been combined with ligament reconstruction in an attempt to give long-lasting correction of both pain and deformity (3).

Lateral calcaneal lengthening osteotomy, as originally described by Evans in child flatfoot was found to restore the medial longitudinal arch and to correct forefoot abduction, thus allowing to minimize the strain and to reach a successful result of the medial ligament reconstruction (4).

The modifications made by Mosca, (1995) on Evans procedure were used in our study; skin incision, direction of the osteotomy, shape of the graft, application of internal fixation and soft tissue procedures respecting the main principles of the original technique lead to successful results in all cases that we performed. 
Our study aims at evaluation of lateral column lengthening with medial soft tissue reconstruction to address both bony and soft tissue abnormalities in symptomatic and significant flexible planovalgus feet in children. This technique was chosen in order to minimize the surgical insult and performing a correction that allows for further growth and remodelling as the osteotomy site is away from growth plate in skeletally immature patients.

The current study was conducted on 29 feet with flexible flat foot deformity [18 male (62\%) and 11 female (38\%)], age ranged from 7 to 17 years (mean; 10.4 years) observed for at least 16 months

The results was at the last follow up 17 patients $(58.6 \%)$ reported complete relief of pain; 12 patients (41.4\%) reported minor occasional pain following surgery. eight patients with mild pain had discomfort at the level of the ankle and subtalar joint. Two patients had discomfort in the calcaneocuboid joint while walking, and another two patients had discomfort in talonavicular joint, but there was no pain when experiencing inversion and eversion.

Patients' evaluation in the current study depends on outcome scoring criteria using American Orthopaedic Foot and Ankle Score, the most commonly used score in the foot and ankle literatures. Our results showed that the mean AOFAS ankle-hindfoot score improved from 43.45 (31-55) preoperatively to 85.52 (68-92) postoperatively at last follow up (P value $<0.001)$.

Radiological improvement of the mean of the lateral talometatarsal angle from $26.9^{\circ}$ preoperative to $3.96^{\circ}$ at last follow up and an improvement of the mean of the $\mathrm{AP}$ talonavicular angle from $32^{\circ}$ preoperative to $7.21^{\circ}$ at last follow up ( $\mathrm{P}$ value $\left.<0.05\right)$.

The results of this study explained the effectiveness of lateral column lengthening as a surgical approach to alleviate pain and deformity resulting from flexible flat foot in children leading to increase their activities at the end of treatment.

Baghdadi T et al (2018) evaluated 30 operated feet of 20 patients who underwent calcaneal lengthening osteotomy, The average age was $10.4 \pm 0.9$ years. Patients were evaluated according to AOFAS score and radiographic indexes. The mean follow up duration was $23.1 \pm 9.9$ months. AOFAS score at the final follow up had improved significantly compared to pre-operative score (37 to 88, $\mathrm{P}<0.0001)$. Radiographic parameters also showed significant improvement after surgery $((\mathrm{P}<0.0001))$. Distal segment displacement and hardware irritation as postoperative complications were observed in 2 and 3 cases, respectively, with no long-term clinical impact (5). 
To evaluate the clinical, functional and radiological outcome of calcaneal lengthening osteotomy for the treatment of symptomatic flatfoot deformity in skeletally immature patients, Marengo et al (2017) assessed 38 operated feet, mean age at time of surgery was $13.3 \pm 2.2$ years (range 7.8-17) AOFAS score improved significantly from a preoperative mean value of $49.9 \pm 16$ (range 23-75) to a postoperative value of $89 \pm 15.9$ (range 34-100) (p < 0.001). All radiographic parameters improved significantly from mean preoperative to mean 3-4-month postoperative value. Correction was maintained at last follow-up visit. Final AP and lateral foot radiographs showed complete bone union and good bone graft remodelling. Furthermore, correct joint alignment was restored in all but two patients (93\%) (6).

Kumar et al (2017) conducted a systematic review on lateral column lengthening for pes planovalgus deformity. They identified seven studies with 103 patients involving 156 feet. The mean age was 13.3 years (Range 5.7-42) and mean follow up was 70.2 Months (Range 24.9156). There was statistical significant improvement in calcaneal pitch, lateral talo-metatarsal and AP talo-navicular angles (p-value 0.001). The mean preoperative AOFAS Score (71 Feet) was 58.85 (Range 34-78) and mean postoperative AOFAS Score (91 Feet) was 92.25 (Range 73-100). Two studies (32 Feet) used author specified criteria has reported Good/Excellent result in 72\% (23/32 feet) and Fair/Poor result in $18 \%$ (9/32) feet. Complications were reported in $17.5 \%$ (18/103) included nerve related, pseudo arthrosis, non-union and metal related complications $(7)$.

\section{Conclusion:}

The results of this study support the combination of lateral column lengthening and soft tissue reconstruction for treatment of Plano-valgus foot deformity with the following advantages:

Correction of all components of the deformity in one sitting, was done. The technique does not use arthrodesis, so the distribution of stresses in the different foot joints is not disturbed. The technique can be done safely in young children as there is no interference with the centres of ossification of the foot bones.. The adopted technique revealed satisfactory result both in children and adolescents with minimal complications.

\section{References:}

1- Mosca V.S. (1995): Calcaneal lengthening for valgus deformity of the hindfoot. J Bone Joint Surg 77-A (4): 500-512.

2- Harris E.J.; Vanore J.V.; Thomas J.L.; Kravitz S.R.; Mendelson S.A.; Mendicino R.W.; et al (2004): Diagnosis and treatment of paediatric flatfoot. J Foot Ankle Surg Nov-Dec; 43(6): 341-73. 
3- Murphy G.A.(2003): Pes planus. In: Campbell's operative orthopaedics, 10th Ed. Mosby Year Bock Inc, Vol. 4, pp.: 4025-4027.

4- Evans DC. (1975): Calcaneo-valgus deformity J Bone JointSurg[Br];57B:270-278.

5- Baghdadi T, Mazoochy H, Guity M, Heidari Khabbaz N. (2018); Evaluation of clinical and radiological results of calcaneal lengthening osteotomy in paediatric idiopathic flexible flatfoot; Arch Bone Jt Surg:402-411.
6- Marengo L, Canavese F, Mansour M, Dimeglio A, Bonnel F.(2017); Clinical and radiological outcome of calcaneal lengthening osteotomy for flatfoot deformity in skeletally immature patients; Eur $\mathbf{J}$ Orthop Surg Traumatol.989-996.

7- Kumar S, and Sonanis SV. (2017); Lateral column lengthening for adolescent idiopathic pes planovalgus deformity: a systematic review; J Orthop;14(4):571-576.

To cite this article: Elshafey A. Elshafey , Mohamed O. Hegazy , Hani A. Bassiouni , Mohamed E. Al-Ashhab. calcaneal lengthening for correction of symptomatic flexible flat foot in children. BMFJ, 2019; 36 (3): 98-106. DOI: 10.21608/bmfj.2020.15010.1021 